\title{
Psicoterapia dinámica breve: Aproximación conceptual y clínica
}

RESUMEN: El objetivo central de este artículo es tratar de esclarecer y discutir, desde un posicionamiento teórico y clínico, las principales características definitorias, las indicaciones y contraindicaciones y los matices técnicos básicos de los diferentes tipos de la psicoterapia dinámica breve, una práctica enraizada en el psicoanálisis que viene mostrando una adecuada eficacia, efectividad y eficiencia en algunos trastornos psíquicos.

PALABRAS CLAVES: Psicoterapia Dinámica Breve, Sifneos, Malan, Davanloo.
SUMMARY: The aim of this paper is to try to clarify and discuss, from a theorical and clinical point of view, the main definitory features, indications and contraindications as well as basic technical details of the different types of shortterm psychodynamic psychotherapy. This form of therapy has its own roots in psychoanalysis having showed an adequate efficacy, effectiveness and efficiency in certain mental disorders.

KEY WORDS: Short-Term Dynamic Psychotherapy, Sifneos, Malan, Davanloo.

\section{Introducción.}

La atención a la salud mental exige en su nivel especializado, tanto en sus compromisos públicos como privados, del manejo de técnicas psicoterapéuticas que posean un amplio campo de acción y que puedan acoger al mayor número posible de demandantes, siempre que cuenten con una probada validez y fiabilidad y se atengan al marco deontológico profesional. En tal sentido, y en lo que se refiere a la perspectiva dinámica, es evidente que el abordaje psicoanalítico estricto sólo está indicado en algunos casos muy concretos, pero las ideas acerca del desarrollo y del funcionamiento psíquicos, las hipótesis de trabajo y la metodología propias del psicoanálisis pueden emplearse, con ciertas modificaciones, para prestar ayuda a sujetos con alteraciones de conducta, síntomas neuróticos o psicóticos y variadas dificultades de adaptación (1). Esto ha abocado a que, en los úúltimos treinta años y algo más recientemente en nuestro país, se vengan imponiendo las técnicas psicoterapéuticas dinámicas breves e intensivas, ya que poseen las características adecuadas para satisfacer las necesidades clínicas de algunos de nuestros pacientes. Tales abordajes pueden competir con la terapia conductual-cognitiva y con la terapia sistémica, por citar las intervenciones más usuales en la actualidad, lo cual es impensable con el uso de la técnica analítica convencional $(2,3,4,5,6)$. Es bien cierto, sin embargo, que no estamos ante ninguna panacea, pues sólo un grupo concreto de demandantes de salud mental pueden beneficiarse de estas intervenciones: estimamos que entre 20-30\% de los pacientes de los circuitos públicos satisfacen los criterios de selección de las terapias dinámicas breves, cifra que se eleva al 50-60\% en los que acceden a las consultas privadas.

Rev. Asoc. Esp. Neuropsiq., 2001, vol XXI, n. ${ }^{\circ} 78$, pp. 7-25 
La psicoterapia dinámica breve no es, en todo caso, una especie de psicoanálisis de saldo, único producto psicoanalítico al que tienen acceso los usuarios de los servicios públicos de salud, dadas sus habituales características socioeconómicas, o los que acceden al circuito privado con pocos medios crematísticos, reservándose el genuino psicoanálisis a la clientela culta y "pudiente". Tal posicionamiento no tiene ninguna fundamentación racional ni de otra naturaleza, ya que la psicoterapia dinámica breve, en cualquiera de sus variantes, supone una práctica genuina que se viene imponiendo en todos los sectores asistenciales, reservándose el análisis clásico para casos muy contados y con indicaciones muy precisas (4).

\section{Delimitación conceptual de la psicoterapia dinámica breve.}

Es preciso diferenciar antes que nada las psicoterapias dinámicas de las terapias de apoyo de orientación psicoanalítica. En tal sentido, destacaremos en primer lugar que éstas tienen su máxima indicación en sujetos psíquicamente muy afectados, que se han descompensado en forma brusca y reciente, así como en familias que han entrado en una crisis de cercana aparición. En cualquiera de los casos, cuando las crisis se prolongan en el tiempo o se dejan evolucionar espontáneamente, los sujetos implicados desarrollan frecuentemente severos trastornos mentales, que se acompañan de graves tensiones en el grupo familiar. De aquí la necesidad de una intervencióón de apoyo pronta e intensiva, con el fin de tratar de aliviar de inmediato la situación psicopatológica activada, mostrando con claridad a los participantes las posibles vías de solución existentes. Son en todo caso son recomendables las siguientes estrategias terapéuticas (7):

1. Ayuda inmediata al sujeto o a la familia en el momento en que la descompensación o la crisis han hecho su aparición, cualquiera que sea la hora del día o de la noche de la demanda, con sesiones de corta duración (unos 30 minutos, aunque en ocasiones pueden prolongarse bastante más, en función de lo que exijan las circunstancias). La primera sesión debe transcurrir en el hogar del paciente, persiguiéndose, antes que nada, la reducción de sus sentimientos de soledad, desvalimiento y desesperanza.

2. Definición del trastorno o del conflicto objeto de la consulta como una crisis, debiéndose incluir en tal delimitación, siempre que sea posible, a todos los miembros del grupo familiar, para evitar que la responsabilidad se sitúe en un único sujeto, defensa que habitualmente se pone en marcha para desviar responsabilidades y culpas.

3. Centrarse en eventos del presente y en los hechos cercanos a la descompensación de la crisis: la toma de la historia clínica, por ejemplo, debe dirigirse a evidenciar los factores inmediatos que han precipitado la descompensación, buscando una formulación dinámica muy superficial, aunque se tengan datos que permitan ir mucho más hondo, pues si se cae en una precipitación ingenua poniendo ante las personas en crisis factores complejos o inconscientes, sólo se logrará el rápido levantamiento de resistencias de muy difícil resolución posterior. 
4. Utilización de medios psicofarmacológicos (por parte del equipo médico), con el fin de controlar lo más inmediata y eficazmente posible la ira, la angustia, la tensión o el pánico que aquejan el o los participantes.

5. Búsqueda de la catarsis en todos los miembros del grupo familiar, responsabilizando (sin acrecentar la angustia o la culpa) a cada uno de ellos en alguna una tarea que contribuya al encuentro de alguna salida positiva a la crisis.

En lo que se refiere a la psicoterapia dinámica breve, hay que resaltar en primer lugar que no se trata de una mera variante de la llamada psicoterapia psicoanalítica, enfoque que inició la escuela de Chicago (8) y que se sigue practicando por parte de algunos terapeutas. Aquí, al margen del problema psicopatológico del sujeto, éste es visto una o dos veces por semana, cara a cara, alentando la libre asociación y ofreciendo interpretaciones dinámicas ocasionales, generalmente centradas en los fenómenos transferenciales más superficiales y más cercanos al yo. En todo caso, se procura ligar las dificultades presentes con los hechos del pasado, especialmente con los de naturaleza inconsciente que se hayan revelado de alguna forma en el curso del proceso terapéutico como significativo. La terapia se extiende durante un año o año y medio, promoviéndose el aumento del autoconocimiento a través de los insights, así como de las imprescindibles experiencias emocionales correctoras (6).

En cuanto a la psicoterapia dinámica breve propiamente dicha, en cualquiera de sus versiones, supone una práctica genuina que nació de la intervención en crisis y del propio psicoanálisis. Respecto a éste, sus apoyos básicos proceden de las aportaciones de Ferenczi, Rank, Reich, Alexander, French, Rosen y los psicólogos del ego (especialmente Pope, Sarvis, Dewees y Johnson). En cuanto a la influencia de la intervención en crisis, hay que mencionar a Lindemann, Tyhurst, Caplan, Quevedo, Bard y Shneidman.

La psicoterapia dinámica breve tampoco es una sofisticada terapia de sostén, sino una forma de proceder específica, en la que se va más allá de los meros reaseguramientos, consejos, simpatía y confrontación con la realidad, como acontece en la mayoría de las terapias de apoyo, suponiendo un trabajo ordenado, sistemático y original, aunque ciertamente sean necesarios los requisitos generales de todo encuentro humano que persiga ayudar psicológicamente, como son la empatía, el respeto y la posibilidad de comunicarse sin emplear ningún juicio desvalorizante de quien solicita nuestra orientación. Pero lo que da un perfil particular y definido a la psicoterapia dinámica breve es el cambio metapsicológico estable del conflicto inconsciente, lo cual habitualmente suele alcanzarse, no pudiendo ser explicado a partir de los ingredientes inespecíficos propios de las terapias de apoyo, sino en función de un marco teórico de naturaleza psicoanalítica y sobre la base de una técnica original claramente definida (9). En efecto, la psicoterapia dinámica breve es una técnica que se muestra muy eficaz para desbloquear el inconsciente, rompiendo las duras resistencias al cambio que muestran muchos neuróticos. 
Una psicoterapia auténticamente científica, como pretende ser la psicoterapia dinámica, debe partir del conocimiento de los factores que originan la conducta inadaptada y poseer algún grado de certeza sobre los medios terapéuticos que han de usarse para modificarla (7). Es decir, ha de caracterizarse por una clara conceptualización de su campo de trabajo, de sus soportes técnicos y de sus objetivos, así como de sus limitaciones. Por ello, nos parece bastante válida la definición que da Barten (10) sobre esta intervención psicoterapéutica: una técnica activa, focalizada, con una meta prevista, circunscrita, cáálidamente mantenida, de acción orientadora y concerniente a las adaptaciones presentes. Nemiah (11), por su parte, le da las siguientes notas: a) acomodación de los conceptos y procedimientos psicoanalíticos a una técnica terapéutica que nos aporta cambios psicológicos significativos en un tiempo menor que el requerido con los métodos clásicos; b) definición de los procedimientos de tratamiento de tal manera que permita enseñarlos rápidamente a otros, además de fijar una serie de firmes criterios de selección de pacientes; $y, c)$ establecimiento de un estudio sobre los abordajes terapéuticos, para verificar científicamente la efectividad de esta psicoterapia.

Un elemento nuclear de la psicoterapia dinámica es, en todo caso, su brevedad, en el sentido de la extensión total del proceso de la cura, que generalmente dura entre 20 y 25 sesiones, todas ellas individuales, no incluyéndose en tal criterio el acortamiento del tiempo de la sesión (por ejemplo, la sesión de veinte minutos de Tedesco o la de diez a doce minutos de Straker), pues entonces sólo se persigue una mayor o menor relegación de los síntomas, esto es, apoyo o sostén, y no cambios psicodinámicos profundos y estables. La mencionada abreviación del proceso de la cura se logra gracias a la concentración del trabajo analítico en determinados focos conflictivos con el uso de elementos técnicos concretos. El foco dinámico o conflictivo quedó bien precisado por Balint, Ornstein y Balint (12), que lo delimitaron como el área particular del problema del paciente que mejor expresa sus dificultades, sus síntomas y su debilidad caracterial (por ejemplo, el estilo de organización de sus defensas) sobre la base de lo cual aparecen habitualmente sus desajustes.

Tras todo lo dicho, estamos en condiciones de caracterizar con mayor precisión los rasgos generales de todo tipo de psicoterapia dinámica breve. Cuatro ingredientes generales identifican estas técnicas: marcada actividad del terapeuta, presencia de elementos técnicos originales, actitud de esperanza y optimismo del terapeuta e interpretaciones tempranas de los fenómenos transferenciales (13).

a) La actividad del terapeuta dinámico es, en efecto, muy marcada y manifiesta, a diferencia de la actuación del analista clásico. El psicoterapeuta dinámico resalta constantemente los focos conflictivos, tratando de mantener la atención del paciente en ellos, llegando incluso a centrar las asociaciones libres en tales áreas. 
b) En cuanto a la originalidad de la técnica, ha de subrayarse que el encuadre viene definido por encuentros cara a cara (jamás se utiliza el diván), con eventual grabación de las sesiones en vídeo con fines de control e investigación. Tras la evaluación inicial, se fija el contrato terapéutico, señalándose la fecha aproximada de la terminación de las sesiones, cuya duración no suele ser tan estricta como la que se da en la técnica estándar, extendiéndose habitualmente en torno a una hora.

Las reglas técnicas básicas del psicoanálisis (asociación libre, atención flotante, neutralidad y abstinencia) son respetadas, pero el proceso terapéutico se trata de acelerar por medio del trabajo en los focos conflictivos más centrales, confrontándose en todo caso las resistencias (defensas) y en concreto los fenómenos transferenciales de manera muy activa, lo que en ocasiones toma el aire de un proceso de naturaleza conductual, incluyendo unas contingencias de control que empujan al sujeto hacia la búsqueda de la salud mental.

c) Respecto a la actitud optimista y esperanzada del terapeuta, ello se verbaliza desde un principio, paráámetro técnico que se ha mostrado como una estrategia útil y eficaz, pues el sujeto encuentra así un clima apropiado para poner en marcha la imprescindible alianza terapéutica y el desarrollo de experiencias emocionales correctivas, aspecto que también asemeja a la psicoterapia dinámica breve a determinadas técnicas cognitivo-conductuales.

d) Finalmente, en lo que apunta al análisis de la transferencia, tradicionalmente se venía manteniendo que ésta no debía tocarse en la psicoterapia breve e intensiva, a lo que se opusieron tanto Sifneos como Malan, demostrando la necesidad y posibilidad de ello si se persiguen cambios psicodinámicos profundos y persistentes y no meras modificaciones sintomááticas o superficiales que desaparezcan pasado un tiempo. Ahora bien, los fenómenos transferenciales se manejan de forma que no evolucionen hacia una neurosis de transferencia, pues ésta se torna una fuente de resistencias de muy difícil disolución.

Tipos de psicoterapia dinámica breve.

Las psicoterapias dinámicas breves acogen diversos tipos de trabajo, con ciertos matices particulares. Al respecto, los principales modelos proceden de Sifneos, Malan y Davanloo.

En cuanto a Sifneos $(15,16)$, su versión de la psicoterapia dinámica (Short-Term Anxiety-Provoking Psychotherapy o STAPP), es aplicable a trastornos con un foco conflictivo central (bien de carácter edípico, bien de pérdida, bien de duelo) que es origen de los más variados síntomas y desajustes, siendo su duración de 6 a 15 sesiones. Ha de señalarse que Sifneos también ha aportado una intervención de apoyo, que en vez de provocadora de ansiedad es supresora de la misma (STASP), procedimiento aplicable 
a sujetos que han tenido importantes problemas en su desarrollo temprano, que son portadores de un yo frágil a débil y que refieren abundantes crisis de descompensación a lo largo de su vida.

Malan $(17,18)$ describe su técnica (Brief Dynamic Psychotherapy o BDP), de unas 20 sesiones en total, como una tarea que persigue la comprensión y el insight de un foco central (edípico, de fracaso o de duelo), así como un cambio en las correspondientes defensas neuróticas, para lograr la mejor conducta adaptativa del sujeto afecto de ciertos trastornos psicopatológicos.

Davanloo $(19,20,21)$, por su parte, incluye el trabajo simultáneo de múltiples focos (Broad-Focus Short-Term Dynamic Psychotherapy o BFSTDP), aceptando a pacientes con muy variada y grave sintomatología (fobias y depresiones crónicas, neurosis obsesivas, diversas caracteropatías, variadas enfermedades psicosomáticas, etc.), habiendo realizado interesantes aportaciones técnicas para ello $(22,23,24,25$, $26,27,28,29,30,31)$.

\section{Evaluaciones de la eficacia.}

Las evaluaciones científicas de las prácticas psicoterapéuticas de apoyo y dináámica han sido y son abundantes. En el caso de las terapias de sostén, por ejemplo, Bellak y Siegel (32) subrayan que el $82 \%$ de los pacientes de su "clínica de paseo" habían mejorado y Langsley y Kaplan (33) también han comprobado, por medio de estudios controlados, el indudable beneficio de las intervenciones en familias, mostrando que los sujetos hospitalizados tras una crisis tratada psicoterapéuticamente necesitaron cinco días de ingreso, frente a los veinticinco días que requirieron los no tratados. Resultados igualmente positivos ha verificado Straker (34) con sus sesiones de veinte minutos.

En lo referente a las psicoterapias dinámicas, Sifneos $(14,15,35,36,37)$ incluyó evaluaciones continuas, investigando el estado de los sujetos al comienzo y al final del tratamiento, constituyéndose los grupos de control por sujetos que entraban en lista de espera y que posteriormente fueron tratados con STAPP. Partiendo de una serie de criterios dinámicos previamente establecidos, dos evaluadores independientes estudiaban la evolución de los sujetos, que habían sido asignados al azar al grupo de tratamiento o al grupo de control. Al finalizar la psicoterapia, generalmente a los cuatro o cinco meses de iniciada, todos los pacientes fueron reevaluados, en cuyo instante los pertenecientes al grupo de control entraban en terapia. Pues bien, los cambios favorables fueron muy evidentes y significativos en las personas tratadas (en torno al $80 \%$ ), aunque Sifneos verificó que el $20 \%$ de los sujetos en lista de espera también habían presentando mejoría de sus síntomas. Sin embargo, cuando éstos fueron entrevistados, resaltaron que en su entorno se habían dado cambios importantes que habían afectado a sus vidas. En cuanto a los sujetos tratados (inicialmente o tras estar un tiempo previo en lista de espera), todos admitieron que la psicoterapia había sido la mejor y más útil experiencia en la resolución de sus conflictos. Un 
año después seguían mostrando capacidad para solucionar adecuadamente nuevos problemas, incluso aquellos pacientes que no habían tenido modificaciones caracteriales profundas. Las investigaciones de Malan (38) han aportado datos similares a los de Sifneos.

A pesar de todo ello, los psicoanalistas freudianos convencionales mantienen que la psicoterapia dinámica breve es un abordaje superficial, que imposibilita la resolución auténtica de las conflictivas más nucleares del sujeto por no alcanzar un correcto análisis de los fenómenos transferenciales, criticando también su alejamiento técnico respecto del genuino psicoanálisis, como ocurre con las duras confrontaciones que habitualmente requiere. Frente a estos ataques, los terapeutas dinámicos presentan sus positivos resultados, con seguimientos de varios años, resaltando que la técnica cláásica, aun con su prolongación en el tiempo y su exhaustivo trabajo de la transferencia, no lleva aparejado un aumento significativo en la eficacia. Tal enfrentamiento entre unos y otros nos parece inadecuado, habida cuenta que ambas técnicas tienen sus indicaciones, no siendo correcto defender que la psicoterapia dinámica breve sea una alternativa que pretenda sustituir al psicoanálisis, sino que es sencillamente un tipo de intervención que puede resolver, con menor tiempo y esfuerzo, muchos trastornos que antes eran, por unas u otras razones, o bien incluidos en el psicoanálisis tradicional o dejados fuera por no cumplir una serie de requisitos. Entendemos, pues, que el análisis-tipo tiene su campo de indicaciones, al igual que la psicoterapia dinámica y las terapias de sostén, aunque ciertamente se den a veces algunas superposiciones, sobre todo entre las dos primeras.

Criterios de selección para acceder a una psicoterapia dinámica breve.

Las indicaciones y contraindicaciones a la psicoterapia dinámica breve han sido bien delimitados y concretados $(2,6)$. Así, para aceptar a un paciente en STAPP, Sifneos $(16,35,36,37)$ establece los criterios siguientes:

1. Existencia en el paciente de un conflicto específico y principal, habitualmente de índole edípica, siendo capaz de seleccionarlo y concentrarse en él.

2. Evidencia de una interrelación del problema presente con tal conflicto nuclear.

3. Habilidad en el sujeto para la comunicación, capacidad para hacer ciertos sacrificios (ser poco narcisista) y aptitud para expresar adecuadamente sus sentimientos (lexitimia).

4. Presencia de un buen nivel intelectual y un cierto grado de sofisticación psicológica, lo que se capta por la forma de responder a las interpretaciones iniciales. Y,

5. Motivación al cambio profundo y no sólo a la relegación de síntomas, lo que puede evaluarse por: a) capacidad para reconocer la clínica de origen psíquico; b) 
predisposición a dar la última razón de los problemas y buen manejo de la introspección; c) habilidad para participar activamente en la terapia; d) predisposición favorable para revivir los conflictos del pasado, curiosidad acerca de sí mismo y expectativas realistas sobre la psicoterapia; y, por último, e) disposición favorable para hacer sacrificios y poseer una mínima disposición para cumplir con las citas y los honorarios.

Malan $(17,18,38)$ ha hecho sus propias especificaciones en cuanto a los criterios de selección de pacientes:

1. Un conflicto actual relativamente circunscrito.

2. Un conflicto nuclear del pasado, más o menos temprano.

3. Una relación congruente entre ambos conflictos.

4. Una adecuada respuesta al test de interpretación efectuado a lo largo de la sesión evaluatoria, de tal manera que tras realizar ciertas interpretaciones, el sujeto aumenta el rapport y el insight de sus problemas.

5. Motivación adecuada al conocimiento de sí mismo.

6. Clara expresión de los conflictos en el campo de la transferencia. Y,

7. Posibilidad de prever una terminación de la terapia y que sus resultados puedan ser dinámicamente explicados en función de los cambios producidos por las intervenciones en el conflicto básico o nuclear.

Otra manera de describir las indicaciones de la psicoterapia dinámica breve (particularmente los modelos de Sifneos y Malan) es por medio de la relación que al respecto da Langsley (7):

1. Psicopatología que no sea severa, sino suave.

2. Reciente aparición del problema que motiva la consulta.

3. Capacidad por parte del paciente de presentar su historia de forma adulta.

4. Síntomas que no se remonten a tiempos muy lejanos ni que se relacionen con problemas psicológicos graves o con una deprivación afectiva.

5. Presencia al menos de una actividad heterosexual a lo largo de la vida anterior.

6. Suficiente motivación a conocerse a sí mismo.

7. Espíritu de cooperación y capacidad para aceptar con gusto las condiciones de la terapia.

8. Respuesta positiva a la prueba de la interpretación. Y,

9. Posibilidad de delimitación de un foco conflictivo concreto en la psicopatología del sujeto.

Como hemos visto, tanto Sifneos como Malan resaltan la importancia de las respuestas del sujeto a las interpretaciones que se efectúan durante la evaluación inicial (test o prueba de la interpretación), cosa que también subrayó Barten (10), el cual manifestó que ello era el indicador más significativo cara ante el pronóstico de la psicoterapia breve. Este autor insistió también en que: 
1. Deben ser excluidos de la psicoterapia dinámica breve los pacientes que manifiesten de forma obstinada las resistencias.

2. Los que nieguen el origen psíquico de sus síntomas o problemas.

3. Los que no puedan mantener un cierto grado de funcionamiento independiente. Y,

4. Los que no pongan con rapidez en marcha una adecuada alianza terapéutica.

Davanloo $(21,39)$, por su parte, ha ampliado las indicaciones de la psicoterapia dinámica breve, señalando que ello no depende del tipo de trastorno que el sujeto arrastre, sino de la forma de desenvolverse en la entrevista evaluatoria inicial, donde han de llevarse a cabo fuertes confrontaciones de las resistencias en las conflictivas de lo que llama vida actual, con el fin de movilizar ira o agresividad en la transferencia, en cuyo momento, en vez de efectuar interpretaciones analíticas, se solicita un relato detallado de las emociones que el sujeto está experimentando entonces. Tras ello, se conectan las conflictivas expresadas en la transferencia con las del pasado reciente y lejano: si todo esto se logra, el paciente se relaja y aumenta su alianza terapéutica, señales evidentes de que es un buen candidato para la terapia dinámica breve, pues tales hechos muestran que es posible desbloquear el inconsciente con facilidad. Dicho de otra manera, el trabajo ha de centrarse en el campo de la resistencia sobre la base de tres criterios: a) desafiar la resistencia como una defensa; b) sacar a la luz los intensos sentimientos transferenciales consecuentes y proporcionar al paciente la posibilidad de experimentarlos; y, c) hacer ver al paciente el paralelismo existente con pautas similares del pasado cercano o lejano. En los casos en que el paciente muestre reacciones ansiosas intensas, ha de enlentecerse el proceso buscando una reorganización de las defensas, para impedir regresiones inconvenientes, dando paso con posterioridad al desbloqueo del inconsciente $(21,22,23)$.

En la entrevista evaluatoria, de hora a hora y media de duración, Davanloo (19, 21) recomienda objetivar una serie de factores, como:

1. Calidad de las interacciones humanas del sujeto y presencia de alguna relación significativa en el pasado.

2. Capacidad para experimentar y tolerar la ansiedad, la culpa y la depresión.

3. Actitud favorable hacia los asuntos psicológicos.

4. Motivación para la introspección y para el trabajo a través de sus descubrimientos, deseo de resolver los problemas y capacidad de insight. Y,

5. Aptitud para responder a las interpretaciones psicoanalíticas.

Aspectos técnicos fundamentales de la psicoterapia dinámica breve.

Los matices técnicos básicos de los tres modelos de psicoterapia dinámica breve, son:

En cuanto a la STAPP, Sifneos $(15,16)$ agrupa sus aspectos técnicos en los siguientes puntos: las sesiones son cara a cara, semanales, de unos 45 minutos de 
duración, con previa especificación de día y hora (que se procurará no modificar), aclarando desde un principio que se trata de una terapia breve, pero sin concretar el número total de sesiones (por lo común la duracióón es de 6 a 15 sesiones). En la sesión evaluatoria (que no realiza el mismo profesional que se hace cargo de la terapia), se concreta si el candidato supera los criterios de selección y se define un foco dinámico, lo que se repite en la primera sesión propiamente terapéutica, recordándole al paciente que debe tratar de concentrarse en el foco conflictivo. Las diversas intervenciones técnicas persiguen los objetivos siguientes (16):

1. Que la alianza de trabajo evolucione y se transforme en alianza terapéutica.

2. Aprovechar tempranamente los sentimientos de transferencia positivos que el paciente experimenta.

3. Mantener una gran actividad terapéutica.

4. Determinar un foco dinámico y mantener el trabajo permanentemente en él.

5. Utilizar reiteradamente las confrontaciones y esclarecimientos que provocan angustia.

6. Enlazar los sentimientos experimentados con personas claves del pasado con lös sentimientos transferenciales.

7. Evitar las regresiones, controlándolas si se ocasionan con el adecuado trabajo técnico.

8. Impedir el desarrollo de una neurosis de transferencia, con las medidas técnicas oportunas.

9. Tratar de poner de manifiesto los nuevos aprendizajes, los modos de resolver problemas y los insights parciales acerca de los conflictos focales que se han logrado en la terapia.

10. En los momentos de resistencia masiva recurrir a las recapitulaciones.

11. Apoyar al paciente cuando su motivación para el cambio es elevada, ayudándolo a superar los sentimientos dolorosos que experimenta.

12. Detectar pruebas tangibles de los cambios de actitud.

13. Demostrar que se ha alcanzado un insight completo de los conflictos que subyacen al foco dinámico.

14. Tratar de dar fin a la terapia en el plazo previsto.

Para profundizar en todo ello, puede consultarse la publicación de Sifneos titulada Short-Term Anxiety-Provoking Psychotherapy. A Treatment Manual (16).

En relación con la intervención propuesta por Malan (18), hay que resaltar, en primer lugar, la necesidad de que en los encuentros terapéuticos impere un clima de aceptación incondicional por parte del profesional, dentro de cuya atmósfera podrá establecerse una interrelación que permita la conscienciación y expresión por parte del paciente de las ideas y sentimientos más ocultos y rechazados. Frente a ellos, el psicoterapeuta debe estar sumamente atento a sus reacciones contratransferenciales, que 
debidamente controladas, y con ayuda del oportuno saber teórico, será el más importante baluarte en que apoyar las oportunas interpretaciones.

Un elemento básico es para Malan el rapport, definido como el grado de contacto afectivo entre paciente y terapeuta (18), lo que a nuestro entender supone el ingrediente esencial de la clásica alianza terapéutica. El estado del rapport debe ser percibido por el terapeuta en cada instante del proceso, pues es la clave que va marcando cómo se desarrolla el mismo: el camino por el cual puede captarse cómo se halla el rapport es observando el grado en que el paciente entra en contacto con sus sentimientos más genuinos y la forma en que se desenvuelve tras una interpretación profunda.

Por otra parte, Malan (18) concreta que la misión del terapeuta dinámico es trabajar sobre la base de dos triángulos, el triángulo del conflicto y el triángulo de las personas. El triángulo del conflicto es algo cercano a lo que Menninger (40) llamó triángulo del insight, implicando enfrentarse sucesivamente a:

1. Los métodos adoptados por los pacientes (defensas) para controlar el dolor psíquico o la angustia que taponan los sentimientos/impulsos ocultos o inaceptables.

2. Las temidas consecuencias (humillación, vergüenza, tristeza, etc.) que traen consigo el conscienciar y expresar tales sentimientos/impulsos. Y,

3. La índole de los contenidos rechazados (sentimientos/impulsos reprimidos).

El triángulo del conflicto (D-A-S/I) se trabaja analíticamente teniendo constantemente en cuenta el triángulo de las personas (T-O-P), constituido por las relaciones que el sujeto tiene con las personas de su vida cotidiana (vértice que Malan llama relaciones con el otro u $\mathrm{O}$ y que Davanloo prefiere etiquetar como relaciones de la vida corriente o $\mathrm{C}$ o de la vida actual o $\mathrm{A}$ ), las relaciones transferenciales que aparecen en el aquí-ahora-conmigo de las sesiones $(\mathrm{T})$ y las relaciones del pasado lejano con los progenitores (P) (véase figura 1). Por otra parte, como puede observarse en la citada figura, hay tres posibles nexos en el triángulo de las personas: el nexo $\mathrm{O} / \mathrm{P}$, en donde existen impulsos y sentimientos rechazados dirigidos a otro $(\mathrm{O})$, que tienen su última procedencia en los que estuvieron referidos a los progenitores (P); el nexo $\mathrm{O} / \mathrm{T}$, que conecta lo que acontece en la vida con el otro (O) con las reacciones transferenciales (T); y el nexo T/P, que liga los deseos expresados ante el terapeuta $(\mathrm{T})$ con los sentimientos/impulsos conflictivos que tuvieron lugar en el pasado infantil respecto los progenitores $(\mathrm{P})$.

En estas representaciones gráficas, Molnos (41) ha realizado una interesante aportación, creando un esquema en el que se considera, en cada uno de los vértices del triángulo de las personas, un triángulo del conflicto. Molnos caracteriza el vértice S/I como X, símbolo que encierra aquello que está oculto y es relevante en el problema del paciente, pudiendo tratarse de un impulso reprimido sexual o agresivo, un dolor 
insoportable, una pena o tristeza, etc. Por otro lado, Molnos da a los tres triángulos internos del conflicto distinto tamaño según su importancia para el problema global y un grosor mayor o menor a sus líneas demarcadoras según la viveza emocional con que el sujeto exprese tales conflictos: así, habitualmente, ha de representarse el tamaño del triángulo del conflicto más grande en el vértice $\mathrm{P}$, luego en el $\mathrm{C}$ y finalmente en el $\mathrm{T}$, ocurriendo lo contrario en cuanto al grosor (véase la figura 2).

La importancia de la innovación de Molnos es que puede permitir la concreción gráfica de la mayoría de las intervenciones terapéuticas, facilitando esto su transmisión y enseñanza. En tal sentido, ha de resaltarse que el saber y la habilidad del terapeuta está en conocer con precisión qué vértice del triángulo de las personas se halla activado en un momento dado del proceso de la cura y analizar en un orden correcto el correspondiente triángulo del conflicto (primero la resistencia/defensa, después el afecto que tapona a los impulsos/sentimientos inconscientes y finalmente éstos, que Molnos llama X), confrontando y disolviendo las oportunas resistencias/defensas, así como elaborando las adecuadas reconstrucciones históricas e interpretaciones y ligando entre sí los distintos vértices del triángulo de las personas, lo que muestra el mayor grado de correlación con la eficacia terapéutica.

Según el enfoque del modelo de Malan (18), el sujeto acude habitualmente a la terapia expresando problemas y dificultades en su vida actual (O). En tal fase de la psicoterapia debe confrontarse, esclarecerse, reconstruirse e interpretarse el conflicto sin salirse de este vértice, siguiendo el orden que antes se ha indicado (D 6 A $6 \mathrm{~S} / \mathrm{I})$. Cuando el contenido $S / I$ (o X)se va situando en la superficie psíquica o acercando al yo, gracias a la debilitación que se ha logrado de la resistencia/defensa (D) y del afecto concomitante (A), es probable que esto provoque un incremento de éste (angustia, cólera, vergüenza, etc.) y subsiguientemente se reactive dicha resistencia/defensa: entonces debe reincidirse en el ataque a tal resistencia hasta que pierda definitivamente su fuerza, lo que suele alcanzarse cuando el paciente puede describir con detalle el concomitante afecto displacentero (angustia, cólera, vergüenza, etc.), cosa que debe efectuarse solicitando primero la expresión de sus ingredientes somatizados y posteriormente los aspectos de índole cognitiva. En tales circunstancias, si el proceso sigue un curso correcto (en lo que la existencia de una potente alianza terapéutica se torna una condición indispensable), los contenidos S/I (X) se van aproximando al yo, pudiendo ser entonces objeto de los oportunos señalamientos y confrontaciones, esclarecimientos, reconstrucciones históricas, interpretaciones y translaboraciones.

Una vez suficientemente dominado el conflicto del vértice $O$, se intentan las debidas conexiones con algunos de los dos vértices restantes del triángulo de las personas. En una terapia en la que la transferencia se desarrolle con lentitud, se elegirá primero el vértice $\mathrm{P}$, estableciendo las oportunas conexiones $\mathrm{O} / \mathrm{P}$, mostrando al sujeto (con apoyo en las confrontaciones, reconstrucciones e interpretaciones) la ligazón pasado-presente que 
se da en sus problemas. En ocasiones, la transferencia es de aparición temprana, en cuyo caso puede iniciarse el trabajo terapéutico en el vértice $T$ y no en el vértice $O$. También puede acontecer que las conflictivas que aparecen en primer plano se refieran a tiempos presentes, pero con los progenitores, sin que apenas se muestren otras temáticas: entonces la terapia sólo se desarrolla en dos vértices del triángulo de las personas (P y T).

Cuando el curso del proceso no se complica, a medida que la terapia avanza, y tras los oportunos análisis de los conflictos en los vértices $\mathrm{O}$ y $\mathrm{P}$, los fenómenos transferenciales van dando la cara cada vez con más intensidad. Tales fenómenos empiezan a manifestarse entonces como resistencias, debiendo actuarse conforme hemos expresado al referirnos al análisis en el vértice $O$ : esto es, tratando de disolver el aspecto resistencial/defensivo (especialmente con el uso de los señalamientos, confrontaciones y esclarecimientos) y luego el afecto concomitante (en base, además de lo anterior, a descripciones de los elementos somáticos y cognitivos de tal afecto), para afrontar finalmente los sentimientos/impulsos más profundos y reprimidos, donde ya se recurre a las interpretaciones profundas. En muchos casos, el conflicto en $T$ será idéntico o muy similar al que acontecía en $O$ ( $y$ cuyo nexo con $P$ ya fue establecido con anterioridad). Esto produce una conexión natural entre $T$ y $\mathrm{O}$, así como entre $\mathrm{T}$ y $\mathrm{P}$. En ocasiones la conexión T/P puede llevarse a cabo directamente, sin la previa inclusión del nexo T/O. Cuando se interpreta el nexo T/P, los sentimientos e impulsos rechazados suelen irrumpir en el yo del paciente con claridad, siendo generalmente evidente la relación de tal material psíquico con la vida infantil y con los progenitores. Si el sujeto toma plena consciencia de esto, la terapia alcanza sus mejores resultados (18).

En el momento en que el proceso terapéutico llega a este punto, esto es, cuando se han ligado los problemas presentes del sujeto con eventos conflictivos del pasado, reales o fantaseados, relacionados con los progenitores o con figuras de especial importancia, y.el paciente ha tomado consciencia de todo esto, puede mencionársele la posibilidad de la próxima terminación del tratamiento, poniendo mucha atención a su reacción, pues es muy probable que este anuncio de la pérdida inmediata del terapeuta reavive antiguas angustias de separación y desengaño con los primitivos objetos de amor. En tal caso, hay que dedicar algunas sesiones a la reelaboración o translaboración de tales sentimientos transferenciales, ligándolos mediante reconstrucciones e interpretaciones a los oportunos hechos del pasado infantil. Sólo entonces puede considerarse que el proceso terapéutico está en condiciones darse por finalizado, lo que suele imponerse de forma natural, conduciendo a la separación de terapeuta y paciente con cordialidad y con sentimientos de alegría por haberse alcanzado la meta prevista. En casos concretos, puede ser necesario retomar al paciente pasado un cierto tiempo, para efectuar algunas sesiones más, puerta que siempre debe dejarse abierta, aunque con el aviso de no retornar a la terapia ante el más mínimo desajuste o sufrimiento, sino sólo cuando se haya comprobado que por sí mismo no pueden resolverse los problemas. 
En el caso de la técnica de Davanloo $(19,20,21)$, aunque se maneja lo esencial de lo descrito, se introducen una serie de variantes, según se actúe con pacientes que presenten un foco edípico, un foco de duelo o múltiples focos. Así, en sujetos con un foco edípico evidente, la característica más importante de la variante es usar fuertes confrontaciones de las resistencias y de los fenómenos transferenciales. Cuando se trabaja con pacientes portadores de focos de duelo o focos múltiples, los cuales suelen estar afectos de una psicopatología severa (fobias u obsesiones crónicas, caracterosis, etc.), Davanloo $(21,22,23,24,25,26,27,28,29,30,31)$ recomienda actuar más lentamente, procurando que se reorganicen ciertas defensas del yo y se establezca una buena alianza terapéutica, después de lo cual puede efectuarse el trabajo de confrontación (42).

Otros aspectos peculiares de la técnica davanlooniana, son: como en el resto de las formas de psicoterapia dinámica, el paciente es visto cara a cara, generalmente una vez por semana, con sesiones de una hora de duración aproximadamente. El sujeto es prontamente informado de que su tratamiento tendrá una duración concreta $(5$ a 15 sesiones en sujetos con foco edípico, 15 , a 25 sesiones para los que evidencien un foco de duelo y 20 a 30 sesiones para los de focos múltiples y patología grave), aunque no es siempre fácil prever el número exacto de sesiones que un determinado sujeto va a necesitar.

Es especialmente típico de la técnica de Davanloo la marcada actividad del terapeuta, con implacables confrontaciones sobre las resistencias en los vértices $\mathrm{C}(\mathrm{o} \mathrm{A})$ y $\mathrm{T}$ del triángulo de las personas, persiguiendo la máás rápida e intensa experimentación por parte del sujeto de los sentimientos ocultos que subyacen en cada conflicto, teniendo esto primacía respecto a las interpretaciones propiamente dichas. Con posterioridad se conectan los sentimientos ocultos en los vértices $\mathrm{C}$ y $\mathrm{T}$, para incluir después el vértice $\mathrm{P}$, todo ello con apoyo en los oportunos esclarecimientos, reconstrucciones $\mathrm{e}$ interpretaciones, buscando insights sobre el por qué se trata de evitar la emergencia de tales afectos. Todo esto permite la puesta en primer plano de los contenidos I/S reprimidos. Ha de subrayarse que no se llevan a cabo reconstrucciones e interpretaciones de tal material en tanto el paciente se esté debatiendo en una resistencia cardinal, volviéndose una y otra vez a ella, hasta su total disolución, lo que suele acontecer tras la detallada descripción del afecto oculto que se pone en primer plano cuando la resistencia ha desaparecido. Ello exige que el sujeto posea capacidad para captar y relatar sus sentimientos (lexitimia).

La neurosis de transferencia se estima como una construcción defensiva, por lo que se confronta e interpreta en cuanto da la cara cualquier atisbo de ella. Ocasionalmente se analiza algún sueño muy significativo, pero esto nunca es un tema de especial relevancia. 
Los resultados terapéuticos son tanto más eficaces cuanto más honda y frecuentemente capta el paciente las interpretaciones que ligan los conflictos expresados en T, C y P, dejándose notar los efectos positivos entre la sexta y octava sesión, lo que se traduce en una mejoría de la adaptación a las circunstancias del presente, en cambios favorables en el carácter y en la mejoría de la relación clínica. Cuando los síntomas empiezan a decaer y el comportamiento se torna progresivamente máás ajustado, debe plantearse la cuestión de la terminación. Ello es bastante fácil en los sujetos con foco edípico, pudiendo necesitar de unas cuantas sesiones extras (de 3 a 5) los casos restantes. En las patologías complejas, que exigen de una mayor prolongación de la psicoterapia para disolver la angustia de separación, el foco terapéutico debe ser tal sentimiento, lo que suele exigir de un duro trabajo terapéutico que enfoque los más enraizados conflictos del pasado.

La técnica de Davanloo ha permitido extender la psicoterapia breve a un número de casos más amplio que los acogidos por las intervenciones de Sifneos y Malan, pudiendo considerarse que el $35-40 \%$ de los sujetos afectos de trastornos psíquicos pueden beneficiarse de este modelo.

Hemos de indicar, por último, que en cualquiera de los tipos de terapia breve, no hay contraindicación respecto a la utilización paralela, al menos durante un cierto tiempo, de ansiolíticos, antidepresivos u otros psicotropos, tratamiento que suele abandonarse a mediados del proceso, bien de forma espontánea por parte del paciente, bien por indicación del terapeuta.

\section{Evaluaciones de la eficacia}

Las evaluaciones científicas de las prácticas psicoterapéuticas de apoyo y dinámica han sido y son abundantes. En el caso de las terapias de sostén, por ejemplo, Bellak y Siegel (36) subrayan que el 82\% de los pacientes de su "clínica de paseo" habían mejorado y Langsley y Kaplan (37) también han comprobado, por medio de estudios controlados, el indudable banaficio de las intervenciones en familias, mostrando que los sujetos hospitalizados tras una crisis trastada psicoterapéuticamente necesitaron cinco días de ingreso, frente a los veintiocho que requirieron los no tratados. Resultados igualmente positivos ha verificado Straker (38) con sus sesiones de veinte minutos.

En los referente a las psitorerapias dinámicas, Sifneos $(14,15,39,40,41)$ incluyó evaluaciones contínuas, investigando el estado de los sujetos al comienzo y al final del tratamiento, constituyéndose los grupos de control por sujetos que entraban en lista de espera y que posteriormente fueron tratados con STAPP. Partiendo de una serie de criterios dinámicos previamente establecidos, dos evaluadores independientes estudiaban la evolución de los sujetos, que habían sido asignados al azar al grupo de tratamiento o al grupo de control. Al finalizar la psicoterapia, generalmente a los cuatro o cinco meses de iniciada, todos los pacientes fueron reevaluados, en cuyo instante los pertenecientes 
al grupo de control entraban en terapia. Pues bien, los cambios favorables fueron muy evidentes y significativos en las personas tratadas (en torno al 80\%), aunque Sifneos verificó que el $20 \%$ de los sujetos en lista de espera también habían presentado mejorías de sus síntomas. Sin embargo, cuando éstos fueron entrevistados, resaltaron que en su entorno se habían dado cambios importantes que habían afectado a sus vidas. En cuanto a los sujetos tratados (inicialmente o tras estar un tiempo previo en lista de espera), todos admitieron que la psicoterapia había sido la mejor y más útil experiencia en la resolución de sus conflictos. Un año después seguían mostrando capacidad pra solucionar adecuadamente nuevos problemas, incluso aquellos pacientes que no habían tenido modificaciones caracteriales profundas. Las investigaciones de Malan (42) han aportado datos similares a los de Sifneos.

A pesar de todo ello, los psicoanalistas freudianos convencionales mantienen que la psicoterapia dinámica breve es un abordaje superficial, que imposibilita la resolución auténtica de las conflictivas más nucleares del sujeto por no alcanzar un correcto análisis de los fenómenos transferenciales, criticando también su alejamiento técnico respecto del genuino psicoanálisis, como ocurre con las duras confrontaciones que habitualmente requiere. Frente a estos ataques, los terapéutas dinámicos presentan sus positivos resultados, con seguimientos de varios años, resaltando que la técnica clásica, aún con su prolongación en el tiempo y su exhaustivo trabajo de la transferencia, no lleva aparejado un aumento significativo en la eficacia. Tal enfrentamiento entre unos y otros nos parece inadecuado, habida cuenta de que ambas técnicas tienen sus indicaciones, no sieneo correcto defender que la psicoterapia dinámica breve sea una alternativa que pretenda sustituir al psicoanálisis, sino que es sencillamente un tipo de intervención que puede resolver, con menor tiempo y esfuerzo, muchos trastornos que antes eran, por unas $u$ otras razones, o bien incluidos en el psicoanálisis tradicional o dejados fuera por no cumplir una serie de requisitos. Entendemos, pues, que el análisis-tipo tiene su campo de indicaciones, al igual que la psicoterapia dinámica y las terapias de sostén, aunque ciertamente se den a veces algunas superposiciones, sobre todo entre las dos primeras. 


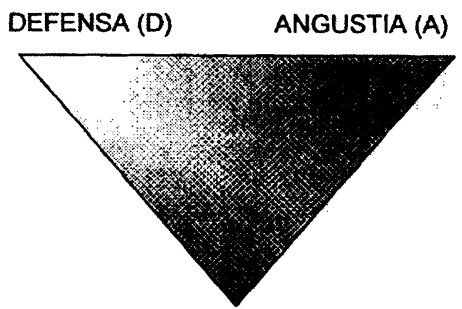

SENTIMIENTO/IMPULSO (S/I)

Triángulo del conflicto

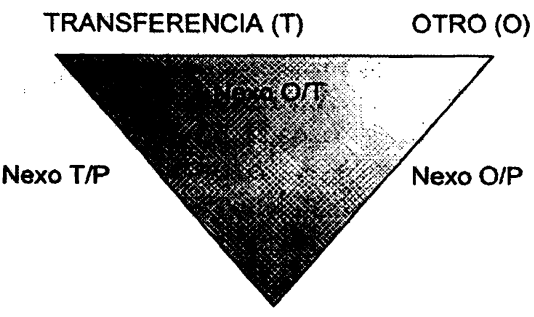

PROGENITORES (P)

Triánqulo de las personas

Figura 1: Los triángulos de Malan

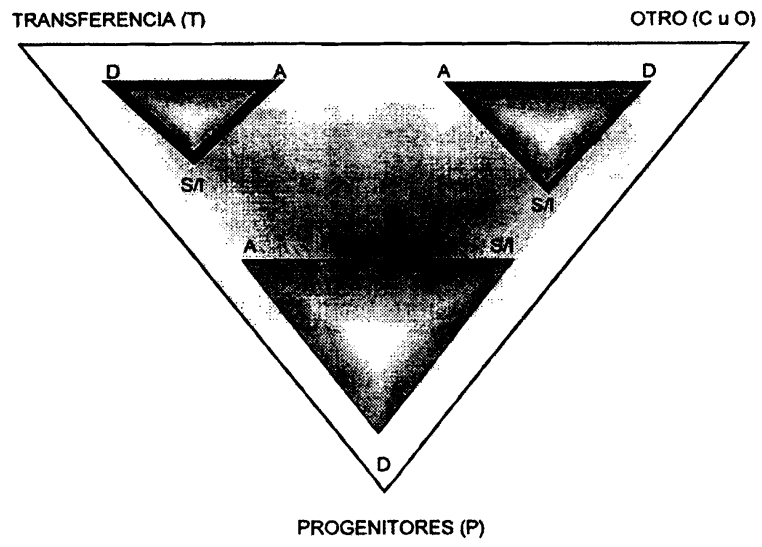

Figura 2: Los triángulos de Molnos 


\section{BIBLIOGRAFÍA}

(1) Coderch, J., Teoría y técnica de la psicoterapia psicoanalítica, Barcelona, Herder, 1987.

(2) Sánchez-Barranco, A., "Psicoterapia breve: criterios de selección y aspectos técnicos", Apuntes de Psicología, 1987, 20-21, 25-30.

(3) Sánchez-Barranco, A., Historia y fundamentos (conceptuales y epistemológicos) de la técnica psicoanalítica, Universidad de Sevilla, Departamento de Psicología Evolutiva y de la Educación, Básica y Metodología, 1989.

(4) Sánchez-Barranco, A., "Técnicas psicoterapéuticas en atención pública: La psicoterapia dinámica breve", en Intervención en salud mental pública, Sevilla, Consejería de Fomento y Trabajo de la Junta de Andalucía, Fondo Social Europeo y Colegio Oficial de Psicólogos (Delegación Andalucía Occidental), 1990.

(5) Sánchez-Barranco, A., Trastorno mental e intervención: enfoque psicoanalítico, Barcelona, Hogar del Libro, 1991.

(6) Sánchez-Barranco, A., Psicoterapia dinámica breve: introducción teórico-técnica, Sevilla, Repiso, 1995.

(7) Langsley, D. G., "Brief Psychotherapy", Journal Canadian Experimental of Psychiatry, dec. 1978, 17-28.

(8) Alexander F. y otros., Terapéutica psicoanalítica, Buenos Aires, Paidós, 1965.

(9) Sánchez-Barranco, A., "Psicoterapia dinámica breve", Apuntes de Psicología, 1983, 5, 8-9.

(10) Barten, H. H., Brief Psychotherapy, New York, Behavioral Publications, 1971.

(11) Nemiah J. C., "Introduction", en D. H. Malan, The Frontier of Brief Psychotherapy, New York, Plenum Press, 1976.

(12) Balint, M., Ornstein, P. H. y Balint, E., Focal Psychotherapy: An Example of Applied Psychoanalysis, London, Tavistock, 1972.

(13) Mendelsohn, R., "Critical Factors in Short-Term Psychotherapy: A Summary", Bulletin of Menninger Clinic, 42, 1978, 42, 133-149.

(14) Sifneos, P. E., "Two Different Kinds of Psychotherapy of Short Duration", American Journal of Psychiatry, 1967, 123, 10-69.

(15) Sifneos, P. E., Short-Term Dynamic Psychotherapy. Evaluation and Technique, $2^{\text {nd }}$ ed., New York, Plenum Press, 1987.

(16) Sifneos, P. E., Short-Term Anxiety Provoking Psychotherapy. A Treatment Manual, New York, Basic Books, 1992.

(17) Malan, D. H., The Frontier of Brief Psychotherapy, New York, Plenum Press, 1976.

(18) Malan, D. H., Psicoterapia individual y ciencia de la psicodinámica, Buenos Aires, Paidós, 1983.

(19) Davanloo, H., Basic Principles and Techniques in Short-Term Dynamic Psychotherapy, New York, Spectrum, 1978.

(20) Davanloo, H., Short-Term Dynamic Psychotherapy, New York, Aronson Inc., 1980.

(21) Davanloo, H., Psicoterapia breve. El desbloqueo del inconsciente, Madrid, Dor, 1992.

(22) Davanloo, H., "Intensive Short-Term Psychotherapy with Highly Resistant Patient. I. Handling Resistance", International Journal of Short-Term Psychotherapy, 1986, 1, 107-133.

(23) Davanloo, H., "Intensive Short-Term Psychotherapy with Highly Resistant Patient. II". Short-Term Psychotherapy, 1986, 1, 239-255.

(24) Davanloo, H., "Intensive Short-Term Psychotherapy with Highly Resistant Depressed Patients. Part I: Restructuring Ego's Regressive Defences", International Journal of Short-Term Psychotherapy, 1987, 2, 99-132.

(25) Davanloo, H., "Intensive Short-Term Psychotherapy with Highly Resistant Depressed Patients. Part II: Royal Road to the Unconscious", International The Course of an Interview after the Initial Breakthrough", International Journal of Short-Term Psychotherapy, 1987, $2,167-185$. 
(26) Davanloo, H., "Clinical Manifestations of Superego Pathology, part I", International Journal of Short-Term Psychotherapy, 1987, 2, 225-254.

(27) Davanloo, H., “Clinical Manifestations of Superego Pathology, part II”, International Journal of Short-Term Psychotherapy, 1987, 3 (1), 1-24.

(28) Davanloo, H., "The Technique of Unlocking of the Unconscious, part I", International Journal of Short-Term Psychotherapy, 1987, 3 (2), 1-121.

(29) Davanloo, H., "The Technique of Unlocking of the Unconscious, part II", International Journal of Short-Term Psychotherapy, 1987, 3 (2), 123-159.

(30) Davanloo, H., "Central Dynamic Sequence in the Unlocking of the Unconscious. Part I: Major Unlocking of the Unconscious", International Journal of Short-Term Psychotherapy, 1988, 4 (1), 1-33.

(31) Davanloo, H., "Central Dynamic Sequence in the Unlocking of the Unconscious. Part II: Major De-repression of the Unconscious", International Journal of Short-Term Psychotherapy, 1988,4 (1), 35-66.

(32) Bellak, L. y Siegel, H., Manual de psicoterapia breve, intensiva y de urgencia, México, Manual Moderno, 1986.

(33) Langsley, D. G. y Kaplan, D. M., The Treatment of Families in Crisis, New York, Grune and Stratton, 1968.

(34) Straker, M. C., "Brief Psychotherapy in an Outpatient Clinic: Evolution and Evaluation", American Journal of Psychiatry, 1968, 124, 35- 75.

(35) Sifneos, P. E., "Seven-year's Experience with Short-Term Dynamic Psychotherapy", en $6^{\text {th }}$ International Congress of Psychotherapy; Selected Lectures, New York, S. Karger, 1965.

(36) Sifneos, P. E., "Learning to Solve Emotional Problems: A Controlled Study of Short-Term Anxiety Provoking Psychotherapy", en R. Porter (comp.), The Role of Learning in Psychotherapy, London, J. and A. Churchill, 1968.

(37) Sifneos, P. E., Short Term Psychotherapy and Emotional Crisis, Cambridge, USA, Harvard University Press, 1972.

(38) Malan, D. H., Toward the Validation of Dynamic Psychotherapy, New York, Plenum Press, 1976.

(39) Davanloo, H., “Techniques of Short-Term Dynamic Psychotherapy”, Psychiatrics Clinics of North America, 1979, 12, 1, 1-22.

(40) Menninger, K. C., Teoría de la técnica psicoanalítica, México, Pax-México, 1966.

(41) Molnos, A., "The two Triangles are Four: A Diagram to Teach the Process of Dynamic Brief Psychotherapy", British Journal of Psychotherapy, 1984, 2, 112-125.

(42) Della Selva, P. C., Intensive Short-Term Dynamic Psychotherapy. Theory and Technique, New York, John Wiley and Sons, Inc., 1996.

* Psiquiatra y psicólogo

** Psiquiatra y psicólogo

Departamento de Psicología Experimental

Universidad de Sevilla

Correspondencia:

Dr. Antonio Sánchez-Barranco Ruiz, Pza. Dr. González Gramage, 2-11B

41005 Sevilla

Fecha de recepción: 1-2-01 